\title{
Local Geometric Proof of Riemann Conjecture
}

\author{
Chuanmiao Chen ${ }^{1,2}$ \\ ${ }^{1}$ School of Mathematics and Statistics, Central South University, Changsha, China \\ ${ }^{2}$ College of Mathematics and Statistics, Hunan Normal University, Changsha, China \\ Email: cmchen@hunnu.edu.cn
}

How to cite this paper: Chen, C.M. (2020) Local Geometric Proof of Riemann Conjecture. Advances in Pure Mathematics, 10, 589-610.

https://doi.org/10.4236/apm.2020.1010036

Received: August 25, 2020

Accepted: October 16, 2020

Published: October 19, 2020

Copyright ( 2020 by author(s) and Scientific Research Publishing Inc. This work is licensed under the Creative Commons Attribution International License (CC BY 4.0).

http://creativecommons.org/licenses/by/4.0/

\section{(c) (i) Open Access}

\begin{abstract}
Riemann hypothesis (RH) is a difficult problem. So far one doesn't know how to go about it. Studying $\zeta$ and using analysis method likely are two incorrect guides. Actually, a unique hope may study Riemann function $\xi(\tau)=u+i v, \tau=\beta+i t, \beta=\sigma-1 / 2$ by geometric analysis, which has the symmetry: $v=0$ if $\beta=0$, and basic expression $v(t, \beta)=-\int_{0}^{\beta} u_{t}(t, r) \mathrm{d} r$. We show that $|u|$ is single peak in each root-interval $I_{j}=\left[t_{j}, t_{j+1}\right]$ of $u$ for fixed $\beta \in(0,1 / 2]$. Using the slope $u_{t}$, we prove that $v$ has opposite signs at two end-points of $I_{j}$. There surely exists an inner point such that $v=0$, so $\{|u|,|v| / \beta\}$ form a local peak-valley structure, and have positive lower bound $\|\xi\|=|u|+|v| / \beta \geq \mu\left(t_{j}, \beta\right)>0$ in $I_{j}$. Because each $t$ must lie in some $I_{j}$, then $\|\xi\|>0$ is valid for any $t$ (i.e. $\mathrm{RH}$ is true). Using the positivity $\operatorname{Re}\left(\xi^{\prime} \mid \xi\right)>0$ of Lagarias (1999), we show the strict monotone $|\xi(t, \beta)|>\left|\xi\left(t, \beta_{0}\right)\right|$ for $\beta>\beta_{0} \geq 0$, and the peak-valley structure is equivalent to RH, which may be the geometric model expected by Bombieri (2000). This research follows Liuhui's methodology: "Computing can detect the unknown and method".
\end{abstract}

\section{Keywords}

Riemann Conjecture, Local Geometric Proof, Symmetry, Peak-Valley Structure, Equivalence, Liuhui's Methodology

\section{Introduction. Difficulty and New Idea}

Riemann conjecture concerns two functions $\zeta(s)$ and $\xi(s)$. In 1737, Euler proved the product formula over all prime numbers $p$ 


$$
\zeta(s)=\sum_{n=1}^{\infty} \frac{1}{n^{\sigma}}=\prod_{p \in \text { prime }}\left(1-\frac{1}{p^{\sigma}}\right)^{-1}
$$

to be convergent for real $\sigma>1$, but divergent for $\sigma \leq 1$. In 1859, Riemann considered the complex variable $s=\sigma+i t, \sigma>1$, using Gamma function $\Gamma(s / 2)$, and got

$$
\zeta(s)=\sum_{n=1}^{\infty} n^{-s}=\pi^{s / 2} \Gamma^{-1}\left(\frac{s}{2}\right) \int_{0}^{\infty} x^{s / 2-1} \psi(x) \mathrm{d} x, \psi(x)=\sum_{n=1}^{\infty} \mathrm{e}^{-n^{2} \pi x} .
$$

Using the functional equality of Jacobi (1828)

$$
2 \psi(x)+1=x^{-1 / 2}\left(2 \psi\left(\frac{1}{x}\right)+1\right)
$$

and transforming the integral by $z=1 / x$

$$
\int_{0}^{1} z^{s / 2-1} \psi(z) \mathrm{d} z=\frac{1}{s(s-1)}+\int_{1}^{\infty} x^{-s / 2-1 / 2} \psi(x) \mathrm{d} x,
$$

Riemann derived the first expression

$$
\zeta(s)=\pi^{s / 2} \Gamma^{-1}\left(\frac{s}{2}\right)\left\{\frac{1}{s(s-1)}+\int_{1}^{\infty}\left(x^{s / 2-1}+x^{-s / 2-1 / 2}\right) \psi(x) \mathrm{d} x\right\},
$$

which is already analytically extended to the whole complex plane except for $s=0,1$. Clearly, the pole points $s=-2,-4,-6, \cdots$ of $\Gamma(s / 2)$ are the trivial zeros of $\zeta(s)$.

Furthermore, Riemann introduced the entire function

$$
\xi(s)=\frac{1}{2} s(s-1) \pi^{-s / 2} \Gamma\left(\frac{s}{2}\right) \zeta(s), \xi(s)=\xi(1-s) .
$$

Through replacing by $\zeta$ and integrating by parts twice, it follows that

$$
\begin{aligned}
\xi(s) & =\frac{1}{2}+\frac{s(s-1)}{2} \int_{1}^{\infty}\left(x^{s / 2-1}+x^{-s / 2-1 / 2}\right) \psi(x) \mathrm{d} x \\
& =r_{1}+\int_{1}^{\infty}\left(x^{s / 2-1}+x^{-s / 2-1 / 2}\right)\left(2 x^{2} \psi^{\prime \prime}+3 x \psi^{\prime}\right) \mathrm{d} x,
\end{aligned}
$$

where $r_{1}=\frac{1}{2}+\psi(1)+4 \psi^{\prime}(1)=0$ derived by (1.2). Riemann had gotten the second expression

$$
\xi(s)=\int_{1}^{\infty}\left(x^{s / 2-1}+x^{-s / 2-1 / 2}\right) f(x) \mathrm{d} x, f(x)=2 x^{2} \psi^{\prime \prime}+3 x \psi^{\prime},
$$

which is symmetric with respect to $s=1 / 2$. He took $\sigma=1 / 2$, then $\operatorname{Im}(\xi)=0$ (I think this is the most important symmetry for $\xi$ ).

Riemann thought that a number of zeros of $\zeta(s)$ in the critical region $\Omega=\{s=\sigma+i t: 0 \leq \sigma \leq 1,0 \leq t<\infty\}$ have an estimate

$$
N(T)=\frac{1}{2 \pi}\left(T \ln \frac{T}{2 \pi}-T\right)+O(\ln T), t \leq T,
$$

(proved by Mangoldt in 1905) then (see our basic theorem and $\S 4.1$ ).

Riemann Hypothesis (RH). All non-trivial zeros of $\zeta(s)$ lie on the critical 
line $\sigma=\frac{1}{2}$.

$\mathrm{RH}$ is a very difficult problem, which has stimulated the untiring research in the areas of the analytic number theory and the complex functions, even the scientific computation. Smale [1] (1998) reported 18 mathematical problems for next century. The first one is RH. Cray Mathematics institute (2000) announced the seven problems of the Millennium, in which RH is reviewed by Bombieri [2]. "Science" (2018) listed 125 science problems, in which 5 mathematics problems include RH.

There have been many theoretical researches for RH, e.g. the reviews [2] [3] and books [4] [5]. A lot of numerical experiments verified that $\mathrm{RH}$ is valid. However, $\mathrm{RH}$ has not been proved to be true or false in theory.

\subsection{Theoretical Research}

We list some important progressions as follows, see [4].

1) Hardy (1914) for the first time proved that $\xi(s)$ has the infinite number of zeros on critical line, by Mellin transform and an important property $\psi\left(z^{2}\right) \rightarrow 0$, as $z \rightarrow \mathrm{e}^{i \pi / 4+0}$. Later Selberg (1942) introduced the correct function near $z^{2}=i$ and proved the number of zeros to be about $c T$ if $0<t \leq T$, where $c \approx 0.01$. Later Levinson (1974) improved with $c=1 / 3$ and Conrey (1989) with $c=2 / 5$. But they are far less than $N(T)$ in (1.7).

2) Poissin (1899) proved no zero of $\zeta$ on line $\sigma=1$, by an interesting equality and singularity decomposition $\zeta(\sigma)=\frac{1}{1-\sigma}+g(\sigma)$ near $\sigma=1$. But it is very hard to extend this conclusion to $\sigma<1$. Up to 1958, Vinogradov and Korobov independently proved no root of $\zeta$ in $\sigma \geq 1-c(\alpha) /(\ln |t|+1)^{\alpha}$, where $\alpha>2 / 3$.

3) The moment method of $\zeta$. Levinson (1975) proved that a number of the roots on critical line attains $34.74 \%$. Conrey (1989) improved to $40 \%$, and then he [3] (2003) pointed out that $99 \%$ of all roots lies in $|\sigma-1 / 2| \leq 8 /|\ln t|$. This is the best result up to now.

4) The Müntz method. A. Durmagambetrov [6] (2016) had proved $|\zeta(s)|>0$, if $\operatorname{Re}(s)>1 / 2+1 / R, 1+\delta<|s|<R$. This is an important progression after Vinogradov and Korobov.

We have seen that except for critical line, most works focus to $\zeta$. We think that the appointing $\zeta$ in formulation of $\mathrm{RH}$ is a historic misguide, because which has gone against the original thinking of Riemann (Actually, he focused to $\zeta$, rather than $\zeta$, see $\S 5$, and $\zeta$ behaves badly, see $\S 6)$. This is likely the first misguide in studying $\mathrm{RH}$.

In recent twenty years, many new methods appeared and the research of RH has taken some important progressions. But RH has not been solved yet.

\subsection{Computational Research}

We can see from (1.7) that the average spacing between two zeros is less than 
$2 \pi / \ln T$. To study the distribution of these zeros, there were lots of large scale numerical experiments, e.g. Lune et al. in [7] [8] searched out $1.5 e+9$ roots on the critical line, where all roots were single, no double. These computations were finished by Euler-Maclaurin formula outside the critical line and Riemann-Siegel formula on the critical line. Here note that Riemann formula (1.6) has not been used! They emphasized that no non-trivial zeros were found in the critical strip $\{0 \leq \sigma \leq 1,0 \leq t \leq 5.6 e+8\}$, which make people have the reason to believe $\mathrm{RH}$ is true. The authors listed lots of computed data and drew many curve figures, which have greatly inspired us to understand the function $\zeta(s)$. There have two surprising phenomena on the critical line.

1) There are a high peak in each segment of the graph and 1 - 9 smaller peaks between two high peaks. They found that the ratio of the high-peak and low-peak can reach 1000 times.

2) There are 1 - 8 roots between two high-peaks. They found a pair of large zeros, these two zeros are very close to each other, and look like a double zero. e.g. in Fig.7, p.678, [8], the spacing between two zeros is less than 0.00011, and the value $Z\left(t^{*}\right)=-0.0000002218$ at the peak point $t^{*}=1048499112.88896415$.

To face so terrible micro-structures near critical line, we has always met a wide gap: how to prove no zeros of the infinite series, analysis method is powerless. Corney [3] (2003) pointed out that "It is my belief, RH is a genuinely arithmetic question that likely will not succumb to methods of analysis". This likely is the second misguide in studying RH. We should give up the infinite summation analysis.

\subsection{A unique Hope is to Study $\xi$}

Although $\zeta$ diverges for $\operatorname{Re}(s) \leq 1$, but can be estimated as follows, see [5] (p.185, 200)

$$
\begin{array}{ll}
|\zeta(\sigma+i t)| \leq C t^{1 / 4-\beta / 2} \ln t, & 0 \leq \sigma \leq 1, \beta=\sigma-1 / 2, \\
\left|\zeta\left(\frac{1}{2}+i t\right)\right|=O\left(t^{\lambda}\right), & \lambda=1 / 6 \text { or } \lambda=19 / 116,
\end{array}
$$

which are possibly expressed in the form

$$
|\zeta(\sigma+i t)| \leq C t^{1 / 6-\beta / 3} \ln t, 0 \leq \beta=\sigma-1 / 2 \leq 1 / 2 .
$$

Denote $\beta=\sigma-1 / 2$. Using an asymptotic expansion

$$
\Gamma\left(\frac{s}{2}\right)=\sqrt{2 \pi}\left(\frac{t}{2}\right)^{\beta / 2-1 / 4} \mathrm{e}^{-t \pi / 4} \mathrm{e}^{i \phi}\left(1+O\left(t^{-1}\right)\right),
$$

(1.4) and (1.10), there has an important estimate with exponential decay [4]

$$
|\xi(s)| \leq C\left(\frac{t}{2}\right)^{23 / 12+\beta / 6} \mathrm{e}^{-t \pi / 4} \ln t \text {, if }|\beta| \leq 1 / 2 .
$$

Due to the decay $\mathrm{e}^{-t \pi / 4}$, it is very hard to compute $\xi(s)$ for large $t$. Probably this is the reason why there are few work to discuss $\xi$. With the same rea- 
son, analysis method is also powerless for $\xi$. Bombieri [2] (2000) pointed out that "We do not have algebraic and geometric models to guide our thinking, and entirely new ideas may be needed to study these intriguing objects". This is a valuable inspiration. We hope to establish a geometric framework.

We know that $\xi$ has the most important symmetry on critical line. We point out that the positivity $\operatorname{Re}\left(\xi^{\prime} / \xi\right)>0$ of Lagarias [9] (1999) is an essential progression for $\xi$, which also is a unique result to be cited in our proof for $\mathrm{RH}$, see §3. Assume that $\mathrm{RH}$ is true, denoting $\xi=u+i v$ and $\tau=\beta+i t, \beta=\sigma-1 / 2$, then

$$
\operatorname{Re}\left(\frac{\xi^{\prime}}{\xi}\right)=\operatorname{Re}\left(\frac{\xi^{\prime} \bar{\xi}}{|\xi|^{2}}\right)=\psi(t) /|\xi|^{2}>0, \text { for } \beta>0
$$

where positive quadratic form

$$
\psi(t)=\xi^{\prime} \bar{\xi}=u u_{\sigma}+v v_{\sigma}=u v_{t}-v u_{t}>0, \text { for } \beta>0 .
$$

plays an important role in our proof.

\subsection{Local Geometric Model for $\xi$}

From these difficulties and advices of Conrey and Bombieri, we should give up $\zeta$ -function and pure analysis methods, while turn to geometric analysis. What is geometric analysis? We no longer regard the summation process of series, while prefer the geometric property and structure of $\xi$-curve itself. That is, "Explain the essence by figure" (Liuhui's words). This is a big change of our recognition. We compute and study by Liuhui thinking, i.e. "computing can detect the unknown and method" (see $\S 7$ ), finally find a local geometric model for $\xi=u+i v$, which contains four basic concepts as follows.

1. Root-interval. For any fixed $\beta \in[0,1 / 2]$, the sub-interval $I_{j}=\left[t_{j}, t_{j+1}\right]$ is called the root-interval, if the real part $u\left(t_{j}, \beta\right)=0, u\left(t_{j+1}, \beta\right)=0$ and $|u(t, \beta)|>0$ inside $I_{j}$.

2. Single peak. If $|u|$ in each root-interval $I_{j}$ only has one peak, called single peak, else called multiple peak (It is proved that the multiple peak case does not exist, see theorem 2).

The single peak $u$ has the following geometry property.

3. Slope $u_{t}$. For single peak $u$ and any $\beta \geq 0$, there are $u_{t}>0$ from negative peak to positive one, and $u_{t}<0$ from positive peak to negative one.

Using Newton-Leibnitz formula, $v(t, 0)=0$ and C-R condition $v_{\beta}=-u_{t}$, we have

4. Analytic property. The imaginary part $v$ has a basic expression

$$
v(t, \beta)=-\int_{0}^{\beta} u_{t}(t, r) \mathrm{d} r, \beta \in(0,1 / 2]
$$

Because $u_{t}$ has opposite signs at two end-points of $I_{j}$, then $v$ also has opposite signs.

Corollary. $|v(t, \beta)| / \beta$ is uniformly bounded with respect to $\beta \in(0,1 / 2]$. 
In numerical experiments we found an important geometry structure as follows.

Peak-valley structure (PVS). For fixed $\beta \in(0,1 / 2]$ and in each root-interval $I_{j}=\left[t_{j}, t_{j+1}\right],|u|$ is a peak. While $v(t, \beta)$ has opposite signs at two end-point of $I_{j}$ and $v=0$ at some inner point, $|v| / \beta$ is a valley. Then $\{|u|,|v| / \beta\}$ form a local PVS and have a local positive lower bound $\|\xi\|=|u|+|v| / \beta \geq \mu\left(t_{j}, \beta\right)>0$ in $I_{j}$ (i.e. $R H$ is valid in $I_{j}$ ).

Using 4 items above, we have proved the PVS (see theorems 1-3).

Sequence principle. As the zeros of u do not have finite condensation point, each $t$ must lie in some $I_{j}$, then $\|\xi\|>0$ is valid for any $t$.

We have gotten

Basic Theorem. All zeros of Riemann $\xi$-function lie on the critical line.

Besides, by (1.12) we have

Equivalence theorem. The peak-valley structure and $R H$ are equivalent.

Theorem 4. The strict monotone $|\xi(t, \beta)|>\left|\xi\left(t, \beta_{0}\right)\right| \geq 0$ for $\beta>\beta_{0} \geq 0$.

We think that the strict monotone is the deepest description for RH. The PVS may be the geometric model to be expected by Bombieri, which makes the proof of $\mathrm{RH}$ get concise and intuitive, and many difficulties are avoided, e.g. need not discuss the summation process of the infinite series and so on.

The PVS and RH in single peak case were shown in our previous paper [10]. This paper wants to give a full proof of RH, including PVS, nonexistence of multiple peak case, the equivalence and strict monotone. I think that I have realized the original thinking line of Riemann, see $\S 5$. Beside we also add the reasons to give up $\zeta$ in $\S 6$. Why I want to study $\mathrm{RH}$ ? My initial aim is to examine that can Liuhui's thinking solve the most difficult problem? which makes me persist in studying in whole four years.

\section{Detect Local Peak-Valley Structure by Computing}

Denote $\tau=i t+\beta, \beta=\sigma-1 / 2$. We consider the Riemann kernel integral $K(f)$ to define

$$
\begin{aligned}
& \xi(\tau)=K(f)=\int_{1}^{\infty}\left(x^{\tau / 2}+x^{-\tau / 2}\right) x^{-3 / 4} f(x) \mathrm{d} x=u+i v, \\
& \xi^{\prime}(\tau)=K^{\prime}(f)=\frac{1}{2} \int_{1}^{\infty}\left(x^{\tau / 2}-x^{-\tau / 2}\right) x^{-3 / 4} \ln x f(x) \mathrm{d} x=u_{\beta}+i v_{\beta}, \\
& \xi^{\prime \prime}(\tau)=K^{\prime \prime}(f)=\frac{1}{4} \int_{1}^{\infty}\left(x^{\tau / 2}+x^{-\tau / 2}\right) x^{-3 / 4} \ln ^{2} x f(x) \mathrm{d} x=u_{\beta \beta}+i v_{\beta \beta},
\end{aligned}
$$

here and below the Cauchy-Riemann conditions are used many times. If $\beta=0$, obviously

$$
x^{i t / 2}+x^{-i t / 2}=2 \cos \left(\frac{t}{2} \ln x\right), x^{i t / 2}-x^{-i t / 2}=2 i \sin \left(\frac{t}{2} \ln x\right),
$$

we have the following analytic property.

The symmetry. If $\beta=0$, then

$$
v=0, u_{\beta}=v_{t}=0, v_{\beta \beta}=-v_{t t}=0, \cdots .
$$


These properties are essential. Especially we have the basic expression (1.12). The norm $|\xi|=\left(|u|^{2}+|v|^{2}\right)^{1 / 2}$ is used in complex analysis. Now define a strong norm

$$
\|\xi\|=\left\{\begin{array}{lll}
|u|+|v| / \beta, & \text { if } \beta \in(0,1 / 2], & t \in[0, \infty) \\
|u(t, 0)|+\left|u_{t}(t, 0)\right|, & \text { if } \beta \rightarrow+0, & t \in[0, \infty)
\end{array}\right.
$$

where three conditions of norm are satisfied. Its advantage is that $|u|$ and $|v| / \beta$ are of the same order and $\|\xi\|$ is stable with respect to $\beta>0$. Note that if $\beta=0, u\left(t_{j}, 0\right)=0, v\left(t_{j}, 0\right)=0$, then $|\xi|=0$, but probably $\|\xi\|>0$, if $\left|u_{t}\left(t_{j}, 0\right)\right| \neq 0$, see Figure 2 .

Firstly we compute $\xi=u+i v$. Take a changing scale $M=8(t / 2)^{23 / 12+0} \mathrm{e}^{-t \pi / 4}$ (where $M$ is independent of $\beta$, different from [10]), when drawing curves of $\xi, x$-axis is $t, y$-axis is $u / M$ such that $|u / M| \leq 1$. No longer explain later. Figure 1 exhibits the curve $u(t, 0)$ and 20 zeros. Note that the zeros of $u$ (real line) and $v / \beta$ (dot line) for $\beta=0.1$ are alternative and have "positive phase-difference", which implies RH. But we do not know how to describe it. After three years, we have suddenly waken up that this is a local PVS.

To explain the local PVS, we consider a smaller root-interval $I_{2}=\left[t_{2}, t_{3}\right]$ in Figure 2 with $\beta=0.1,0.3,0.5$. We see that $u\left(t_{2}, \beta\right)=0, u\left(t_{3}, \beta\right)=0$ at two end-points of $I_{2}$, and $u(t, \beta)>0$ inside $I_{2}, u$ is a positive peak. We also see that $v\left(t_{2}, \beta\right)<0, v\left(t_{3}, \beta\right)>0$, and $v\left(t_{2}^{\prime}, \beta\right)=0$ at some inner point $t_{2}^{\prime} \in I_{2}$, $|v| / \beta \geq 0$ is a valley. Then $\{|u|,|v| / \beta\}$ form a local PVS in $I_{2}$ and there is the lower bound $\min _{t \in I_{2}}(|u|+|v| / \beta) / M \geq 0.0876$, i.e. $\mathrm{RH}$ is valid in $I_{2}$.

Nextly we compute the derivative $i \xi^{\prime}=i\left(u_{\beta}+i v_{\beta}\right)=u_{t}+i v_{t}$. We see that $u_{t}$ and $v_{t}$ have also alternative zeros and a local PVS in Figure 3.

\section{Local Geometric Proof of RH}

We regard $\{u(t, \beta), v(t, \beta)\}$ as a continuous changing process from $\beta=0$ to
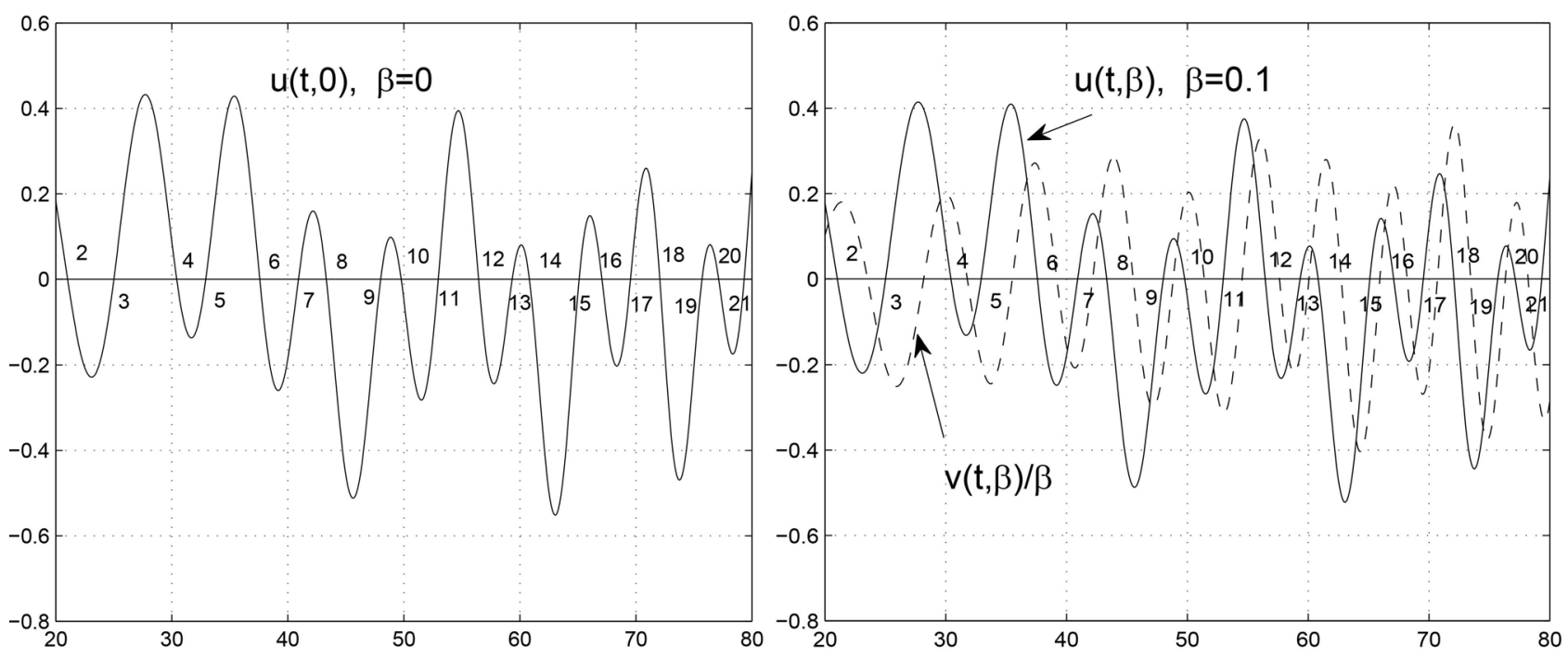

Figure 1. Left: $u(t, 0)$. Right: $u$ (real line) and $v / \beta$ (dot line) for $\beta=0.1$. 

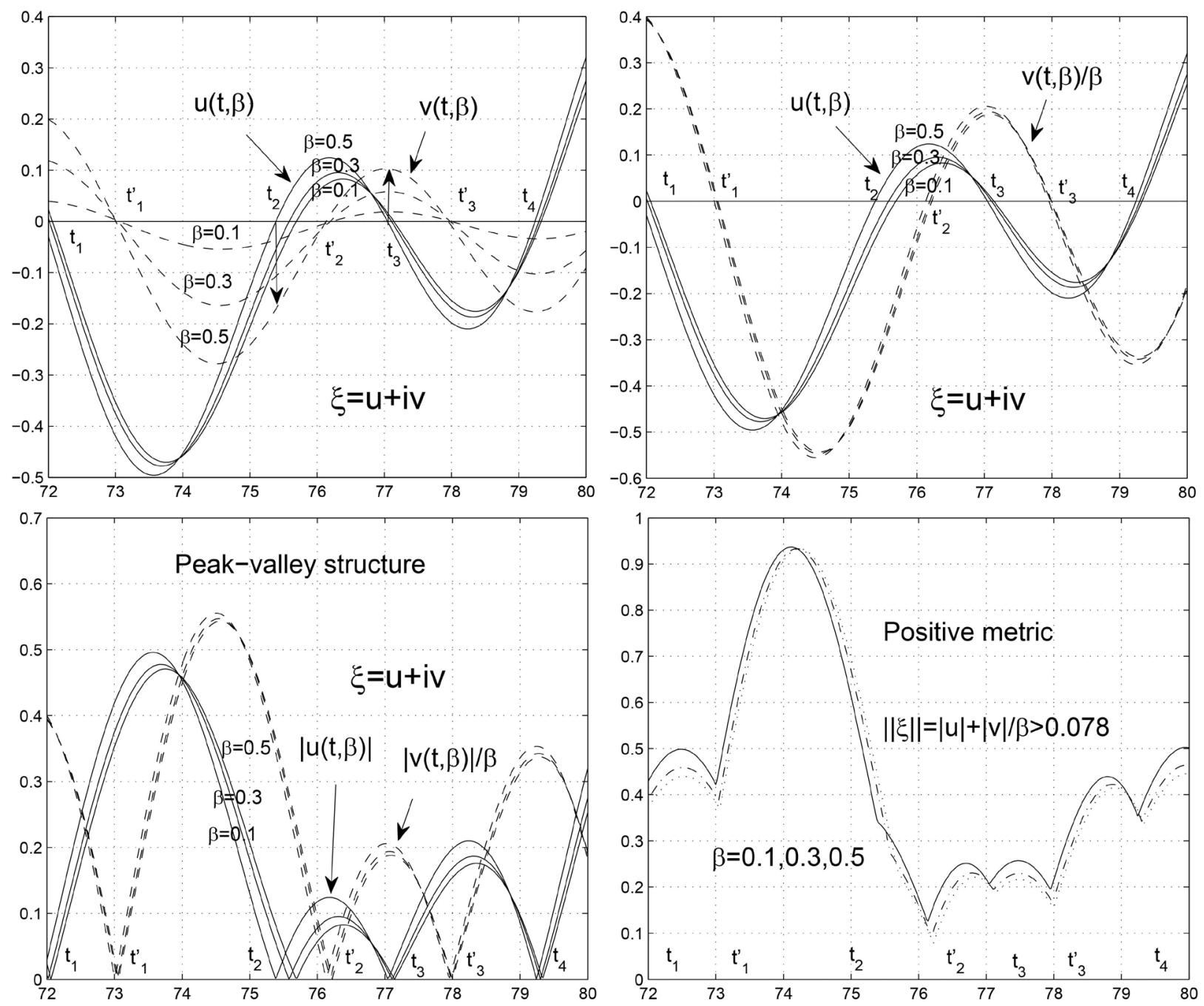

Figure 2. Curves $\{u, v\},\{u, v / \beta\},\{|u|,|v| / \beta\}$ and $|u|+|v| / \beta>0$.
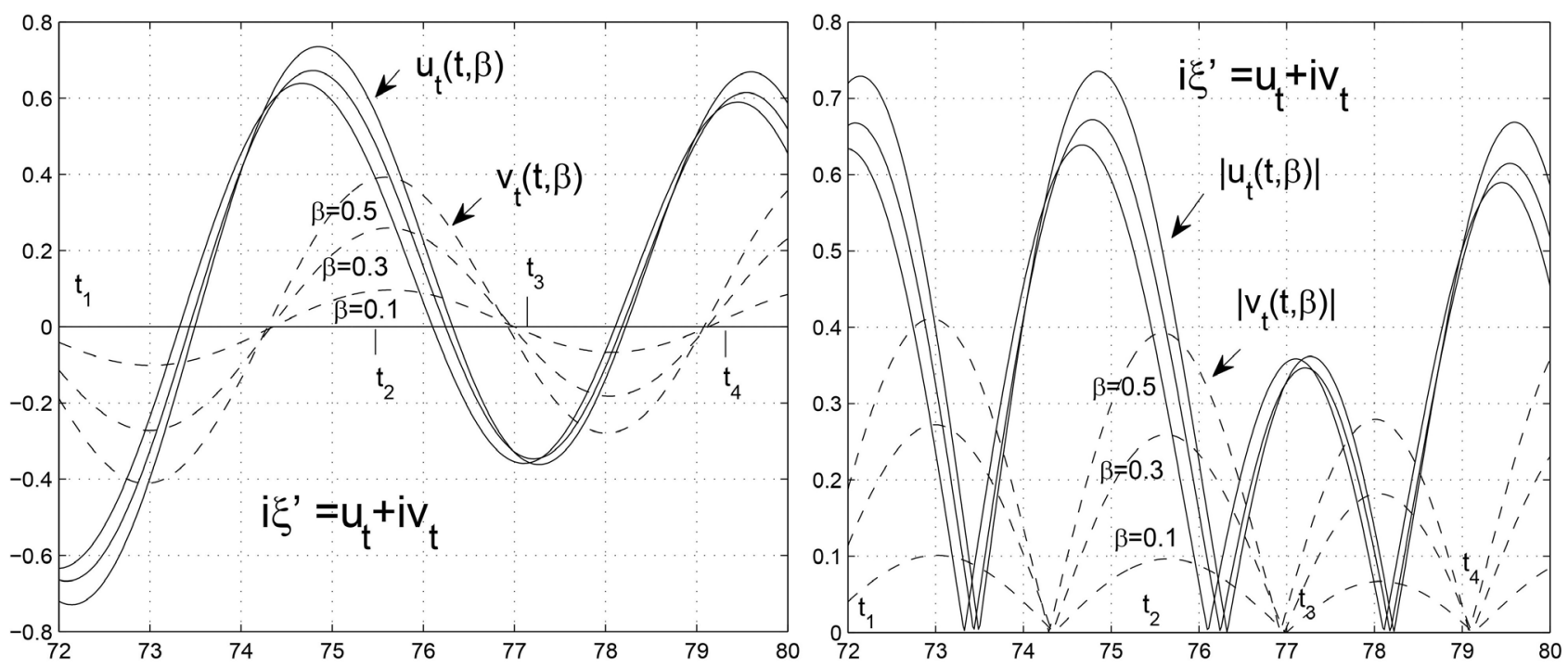

Figure 3. Curves $\left\{u_{t}, v_{t}\right\}$ and $\left\{\left|u_{t}\right|,\left|v_{t}\right|\right\}$, compare with Figure 2. 
$\beta=1 / 2$. For any $\beta \in(0,1 / 2]$, all zeros $t_{j}$ of $u(t, \beta)$ form an irregular infinite sequence dependent on $\beta$

$$
0<t_{1}<\cdots<t_{j-1}<t_{j}<t_{j+1}<t_{j+2}<\cdots \rightarrow \infty,
$$

which have not the finite condensation points, else $u \equiv 0$. We take them as the base in studying PVS.

Theorem 1 (single peak case). If $u(t, \beta)$ is single peak for any $\beta \in(0,1 / 2]$, then $\|\xi\|>0$ for any $(t, \beta) \in \Omega=[0, \infty) \times(0,1 / 2]$.

Proof. Below it is enough to discuss $u>0$ inside the root-interval $I_{j}=\left[t_{j}, t_{j+1}\right]$. For any fixed $\beta>0$, using the analytic property (1.12), we consider two cases as follows.

As $u_{t}>0$ near the left node $t_{j}$, we have

$$
\left\{\begin{array}{l}
v\left(t_{j}, \beta\right) / \beta=-\frac{1}{\beta} \int_{0}^{\beta} u_{t}\left(t_{j}, r\right) \mathrm{d} r<0, \\
\lim _{\beta \rightarrow+0} v\left(t_{j}, \beta\right) / \beta=-u_{t}\left(t_{j}, 0\right)<0 .
\end{array}\right.
$$

As $u_{t}<0$ near the right node $t_{j+1}$, similarly

$$
\left\{\begin{array}{l}
v\left(t_{j+1}, \beta\right) / \beta=-\frac{1}{\beta} \int_{0}^{\beta} u_{t}\left(t_{j+1}, r\right) \mathrm{d} r>0, \\
\lim _{\beta \rightarrow+0} v\left(t_{j+1}, \beta\right) / \beta=-u_{t}\left(t_{j+1}, 0\right)>0 .
\end{array}\right.
$$

They are valid and numerically stable for $\beta \in(0,1 / 2]$.

Because $v(t, \beta)$ has opposite signs at two end-points in $I_{j}$, there certainly exists an inner point $t_{j}^{\prime}=t_{j}^{\prime}(\beta)$ such that $v\left(t_{j}^{\prime}, \beta\right)=0$. Clearly in $I_{j},|u|$ is a peak and $|v(t, \beta)| / \beta$ is a valley, thus $\{|u|,|v| / \beta\}$ form a local PVS. We regard $\|\xi(t, \beta)\|$ as a continuous function of $(t, \beta)$, which certainly has a positive lower bound independent of $t \in I_{j}$,

$$
\min _{t \in I_{j}}\|\xi(t, \beta)\|=\mu\left(t_{j}, \beta\right)>0, \beta \in(0,1 / 2] .
$$

This is a fine local geometric analysis.

Thus in each root-interval $I_{j}=\left[t_{j}, t_{j+1}\right]$, we can determine a positive lower bound $\mu\left(t_{j}, \beta\right)>0$, which form the positive infinite sequence

$$
\mu\left(t_{1}, \beta\right), \mu\left(t_{2}, \beta\right), \cdots, \mu\left(t_{j}, \beta\right), \mu\left(t_{j+1}, \beta\right), \cdots
$$

Because each $t$ must lie in some $I_{j}$, thus $\|\xi\|>0$ for any $t$. In this way, the summation process of the infinite series $\xi$ is completely avoided.

Theorem 2. The multiple peak case does not exist for $\beta \in[0,1 / 2]$.

Proof. Assume that $u(t, \beta)>0$ for $\beta>0$ inside some root-interval $I_{j}=\left[t_{j}, t_{j+1}\right]$ and has odd number of extreme values $u\left(t_{j p}, \beta\right)=a_{j p}>0$ at the inner points $t_{j p}, p=1,2, \cdots, 2 k+1$, see Figure 4. Clearly, $u>0$ in $I_{j}^{\prime}=\left[t_{j 1}, t_{j, 2 k+1}\right]$, while in two sub-intervals $\left[t_{j}, t_{j 1}\right]$ and $\left[t_{j, 2 k+1}, t_{j+1}\right]$ we have (3.1) and (3.2). So $\|\xi\|>0$ in $I_{j}$ and RH is still valid for any $t$.

Below we prove that the multiple peak case does not appear. For this, we consider the minimum extreme value $u\left(t_{j i}, \beta\right)=a_{j i}>0$ at some point $t^{\prime}=t_{j i}$, in 


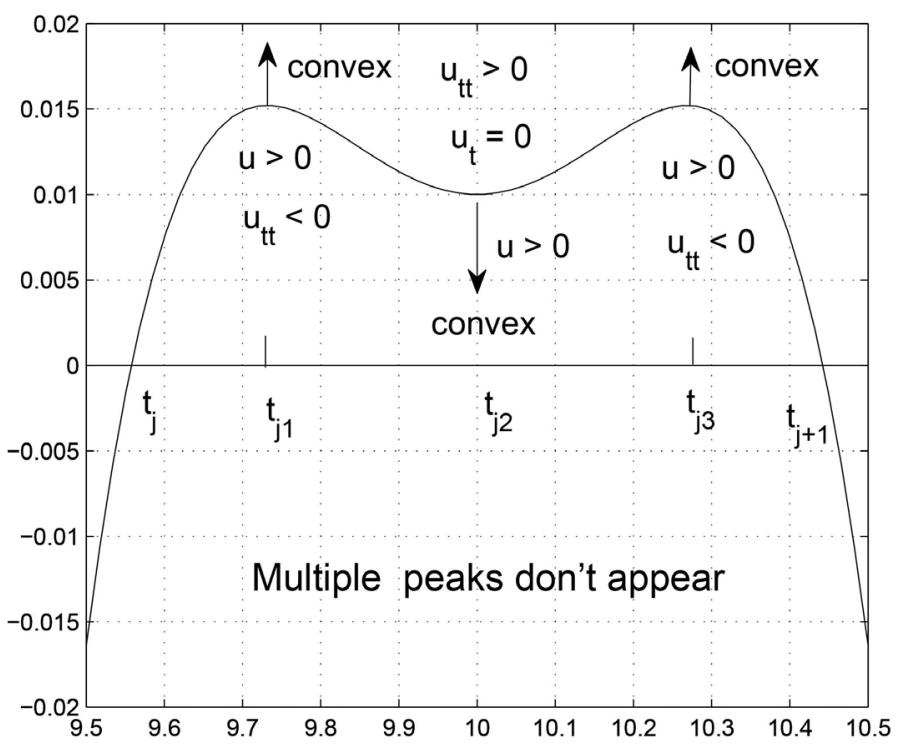

Figure 4. Different convexities.

there $u_{t}=0$ and $u_{t t}>0$, i.e., $u$ is locally convex downwards, see Figure 4. By basic expression we have

$$
v_{t}\left(t^{\prime}, \beta\right)=-\int_{0}^{\beta} u_{t t}\left(t^{\prime}, r\right) \mathrm{d} r<0 .
$$

On the other hand, because RH is valid as before, using (1.12), we have

$$
\psi(t)=u v_{t}-v u_{t}>0, \beta>0 .
$$

But now, $u>0, u_{t}=0, v_{t}<0$ at $t=t^{\prime}$, which lead to contradiction $\psi=u v_{t}<0$. Thus $u(t, \beta)$ for any $\beta>0$ is single peak and its limit $u(t, 0)$ is also single peak.

Why need to deny the multiple peak case? We see in Figure 4 that when $\beta$ grows, the curve $u(t, \beta)>0$ near $t=t_{j 2}$ will decrease towards its local convex direction (see proof in theorem 3 ). This will bring a dangerous possibility to be close to $t$-axis such that $u=v=0$.

Theorem 3. The peak curve $u(t, 0)$ in a small root-interval (including double root) will remove in parallel towards its convex direction for $\beta>0$ so that $\|\xi(t, \beta)\|>0$.

Proof. Assume that $u(t, 0)$ has a solitary small root-interval $I_{j}^{0}=\left[t_{j}^{0}, t_{j+1}^{0}\right]$ such that $u\left(t_{j}^{0}, 0\right)=0, u\left(t_{j+1}^{0}, 0\right)=0$, and $u(t, 0) \geq 0$ in $I_{j}^{0}$. Let the maximum value $u\left(t^{\prime}, 0\right)=\epsilon>0$ at some inner point $t^{\prime} \in I_{j}^{0}$, then $u_{t}\left(t^{\prime}, 0\right)=0$ and $u_{t t}\left(t^{\prime}, 0\right)<0$. Consider a little enlarged sub-interval $I \supset I_{j}^{0}$, in which $u_{t t}(t, 0)<0$ and $u(t, 0) \leq \epsilon$ is convex upwards. See Figure 5. So $u_{t}>0$ for $t<t^{\prime}$ and $u_{t}<0$ for $t>t^{\prime}$. Note that double zero $u\left(t^{\prime}, 0\right)=\epsilon=0$ is admissible.

Take a small $\beta>0$. By basic expression in $I$ we have

$$
v_{t}(t, \beta)=-\int_{0}^{\beta} u_{t t}(t, r) \mathrm{d} r>0, \text { as } u_{t t}(t, r)<0,
$$

and 


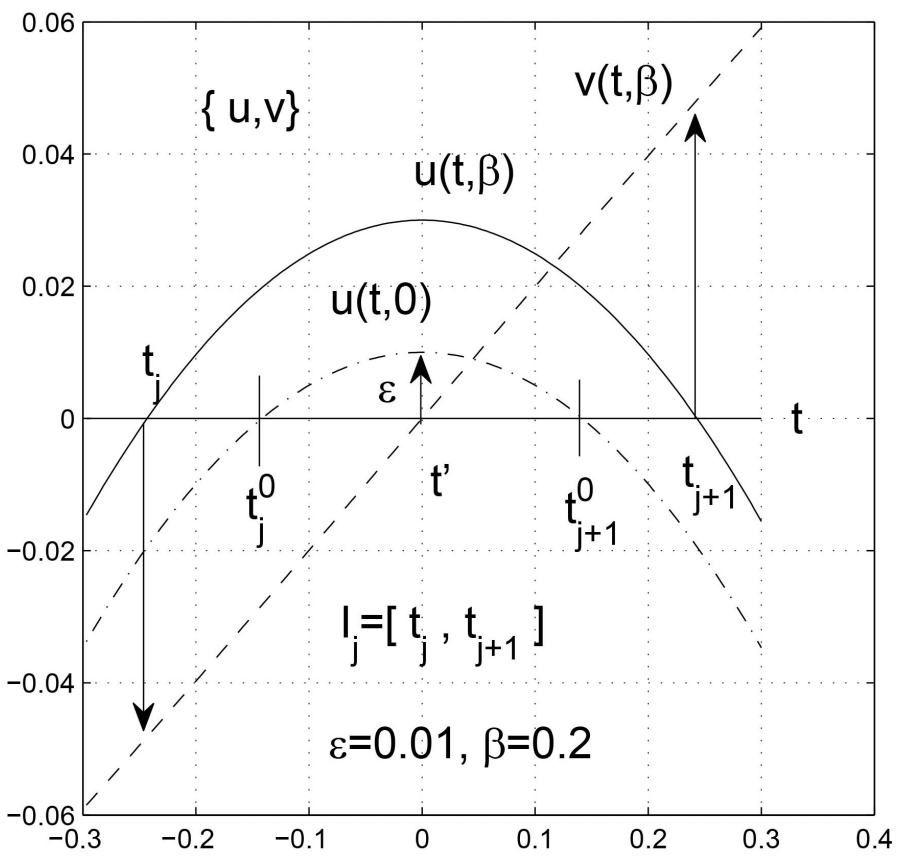

Figure 5. Artificial example $(u, v)$.

$$
\begin{aligned}
u(t, \beta)-u(t, 0) & =\int_{0}^{\beta} u_{\beta}(t, r) \mathrm{d} r=\int_{0}^{\beta} v_{t}(t, r) \mathrm{d} r, \text { as } u_{\beta}=v_{t}, \\
& =-\int_{0}^{\beta}\left(\int_{0}^{r} u_{t t}\left(t, r^{\prime}\right) \mathrm{d} r^{\prime}\right) \mathrm{d} r=d>0 .
\end{aligned}
$$

So $u(t, \beta)$ has removed $u(t, 0)$ in parallel upwards by a distance $d>0$ (i.e. towards its convex direction).

Note that $u(t, 0)<0$ outside $I_{j}^{0}$. For $\beta>0, u(t, \beta)$ has already removed upwards by $d>0$, so there surely exists an enlarged sub-interval $I_{j}=\left[t_{j}, u_{j+1}\right] \supset I_{j}^{0}$ such that,

at the left node $t_{j}=t_{j}(\beta)<t_{j}^{0}, u\left(t_{j}, \beta\right)=0$ and $u_{t}\left(t_{j}, \beta\right)>0$,

at the right node $t_{j+1}=t_{j+1}(\beta)>t_{j+1}^{0}, u\left(t_{j+1}, \beta\right)=0$ and $u_{t}\left(t_{j+1}, \beta\right)<0$,

i.e. $I_{j}=\left[t_{j}, t_{j+1}\right] \supset I_{j}^{0}$ is new root-interval of $u(t, \beta)$, and $u(t, \beta)>0$ inside $I_{j}$, see Figure 5 .

Besides, by basic expression and the slope $u_{t}$, we know

$$
v\left(t_{j}, \beta\right)<0\left(\text { as } u_{t}>0\right), v\left(t_{j+1}, \beta\right)>0\left(\text { as } u_{t}<0\right) .
$$

So there surely exists an inner point $t_{j}^{*} \in I_{j}$ such that $v\left(t_{j}^{*}, \beta\right)=0$, i.e., $|v| / \beta$ still is a valley. Therefore $\{|u|,|v| / \beta\}$ in $I_{j}$ form a local PVS and $\|\xi\|>0$ is valid in $I_{j}$.

Lune et al. [8] pointed out that all roots on critical line are single, no double. Maybe, in the future, some double roots are found, but in this case, theorem 3 still confirms $\|\xi\|>0$ for $\beta>0$. This is one of the most mysterious property for $\xi$.

Summarizing three theorems above, our basic theorem is proved.

Remark. In the proof of Theorem 1 we have seen that the Riemann integral $\xi=K(f)$ has the symmetry, which is independent of the speciality of $f$. So we 
guess that for the very wide class of the fast decay function $f$, RH is still valid for $K(f)$. We have two examples. For $t \leq 110$, there are larger low bounds $\left(\left|u_{\beta}\right| / \beta+\left|v_{\beta}\right|\right) / M \geq 0.20$ and $\left\|\xi^{\prime \prime}\right\| / M \geq 0.28$.

Haglund [11] has discussed $\Xi$ and other functions with numerical experiments, and proposed a conjecture: If function $F_{N}$ has monotonic zeros, then which implies RH. Sarnak [12] has analyzed the Grand RH of L-function, which are more difficult.

\section{Lagarias Theorem and Other Conclusions}

In the proof of $\mathrm{RH}$, we have used a unique new result to be the following.

Lagarias theorem (1999). If $R H$ is true, then $\operatorname{Re}\left(\frac{\xi^{\prime}(\tau)}{\xi(\tau)}\right)>0$ for any $\beta>0$.

This is a unique equivalence to $\mathrm{RH}$ for $\xi$, we think that this is an essential progression along research line of $\xi$ after Hadamard (1893) and Mongoldt (1905), which cannot be directly derived from the integral form (1.6). The multiplication and division operations for the integral form of $\xi$ are impossible.

A simplified proof. If RH is true, Hadamard (1893) proved a product expression

$$
\xi(s)=\mathrm{e}^{A+B s} \prod_{\rho}\left(1-\frac{s}{\rho}\right) \mathrm{e}^{s / \rho},
$$

here $\rho$ runs over all roots of $\xi(\rho)=0$, and $A$ and $B$ are some constants.

Now, we transform $\tau=s-1 / 2=\beta+i t$, their roots are conjugate, $\tau_{j}=i t_{j}, \bar{\tau}_{j}=-i t_{j}$, i.e. $\tau / \tau_{j}+\tau / \bar{\tau}_{j}=0$. All positive zeros $t_{j}$ form an infinite series

$$
0<t_{1}=14.134<t_{2}<t_{3}<\cdots<t_{j}<\cdots
$$

Lune et al [8] pointed out that all zeros are single, no double. But below double zeros are admissible. Then it is simplified to

$$
\xi(\tau)=\mathrm{e}^{A+B \tau} \prod_{j}\left(1-\frac{\tau}{\tau_{j}}\right)\left(1-\frac{\tau}{\bar{\tau}_{j}}\right), \tau=\beta+i t .
$$

If $\beta=0, \xi$ is real, then $B=0$. So we get a product expression

$$
\xi(\tau)=\xi(0) \prod_{j=1}^{\infty}\left\{\left(1-\frac{\tau}{\tau_{j}}\right)\left(1-\frac{\tau}{\bar{\tau}_{j}}\right)\right\}, 0<\beta \leq 1 / 2,0 \leq t<\infty .
$$

Taking logarithm and derivation, we have

$$
\frac{\xi^{\prime}}{\xi}=\sum_{j=1}^{\infty}\left\{\frac{1}{\tau-\tau_{j}}+\frac{1}{\tau-\bar{\tau}_{j}}\right\}=\sum_{j=1}^{\infty}\left\{\frac{\beta-i\left(t-t_{j}\right)}{\beta^{2}+\left(t-t_{j}\right)^{2}}+\frac{\beta-i\left(t+t_{j}\right)}{\beta^{2}+\left(t+t_{j}\right)^{2}}\right\},
$$

then

$$
\operatorname{Re}\left(\frac{\xi^{\prime}}{\xi}\right)=\beta \sum_{j=1}^{\infty}\left\{\frac{1}{\beta^{2}+\left(t_{j}-t\right)^{2}}+\frac{1}{\beta^{2}+\left(t_{j}+t\right)^{2}}\right\}>0 .
$$


It remains to explain its convergence. In fact, by the estimate $N(T) \approx \frac{T}{2 \pi} \ln \left(\frac{T}{2 \mathrm{e} \pi}\right)$, the average spacing between two adjacent zeros is about $2 \pi / \ln T$. For $j$ suitably large, the zero point $t_{j}$ has an approximate estimate

$$
t_{j} \approx(j \ln j) / 2 \pi \text {. }
$$

Thus for any fixed $t \geq 0$, the series is convergent.

\section{Proof of strict monotone.}

Denote $\xi=u+i v$, using the positive quadratic form $\psi=u u_{\beta}+v v_{\beta}>0$, we have

$$
|\xi(t, \beta)|^{2}-\left|\xi\left(t, \beta_{0}\right)\right|^{2}=2 \int_{\beta_{0}}^{\beta}\left(u u_{\beta}+v v_{\beta}\right) \mathrm{d} \beta>0, \quad \beta>\beta_{0} \geq 0
$$

then $|u(t, \beta)|>\left|u\left(t, \beta_{0}\right)\right|$.

Figure 6 exhibits the positivity $\operatorname{Re}\left(\xi^{\prime} / \xi\right)>0$ and the strict monotone for $\beta=0.1,0.3,0.5$. Note that in there the changing scale $M=8(t / 2)^{23 / 21+0} \mathrm{e}^{-t \pi / 4}$ should be independent of $\beta$. (Note that if take $M(\beta)=8(t / 2)^{23 / 21+\beta / 6} \mathrm{e}^{-t \pi / 4}$, we find that $|\xi(\tau)| / M(\beta)$ does not have the monotone with respect to $\beta)$ !

\section{Proof of the equivalence theorem.}

Assume that $\mathrm{RH}$ is valid and $u>0$ inside root-interval $I_{j}=\left[t_{j}, t_{j+1}\right]$ (similarly for $u<0)$. By Lagarias theorem, the quadratic form $\psi=u v_{t}-v u_{t}>0$ in $I_{j}$ for $\beta>0$, and geometric property of $u_{t}$, we have the following facts.

At the left node $t_{j}, u=0, u_{t}>0$ and $\psi=-v u_{t}>0$, then $v<0$;

At the right node $t_{j+1}, u=0, u_{t}<0$ and $\psi=-v u_{t}>0$, then $v>0$.

Thus $v$ has opposite signs at two end-points, there certainly exists an inner point $t_{j}^{\prime} \in I_{j}$ such that $v=0$, which implies local PVS. Thus the equivalence of both is proved.
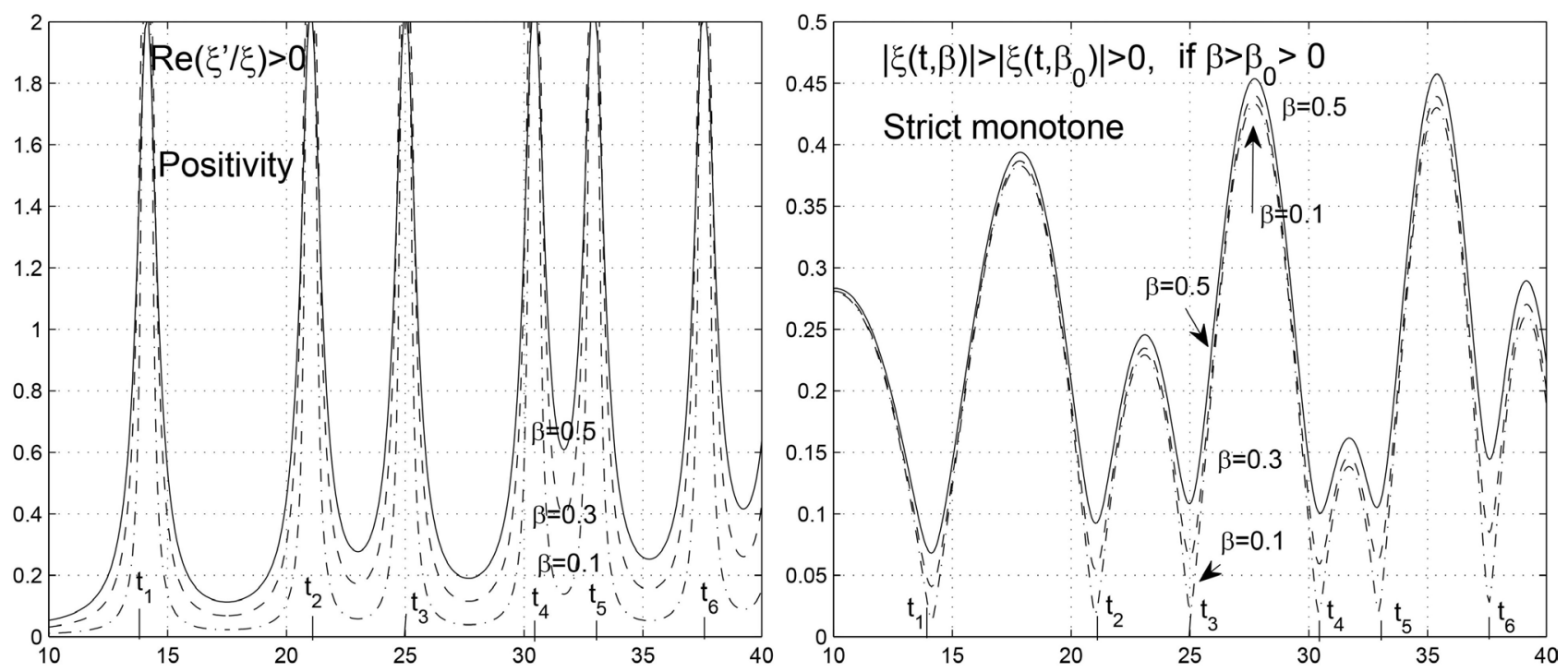

Figure 6. Positivity and strict monotone $|\xi(t, \beta)|>\left|\xi\left(t, \beta_{0}\right)\right|>0$ if $\beta>\beta_{0}>0$. 
From the view-point of complex analysis, RH requires $|\xi|>0$, while from the view-point of geometry, the peak-valley structure requires strong norm $\|\xi\|>0$. Both are equivalent. However, the local geometry property is of extreme importance, which makes the proof be concise and intuitive. I greatly appreciate the mathematical beauty of the symmetry.

\section{Follow Riemann Thinking}

Riemann's paper "On the number of primes less than a given magnitude" is a classic work [5], we consider a part of it, and give remarks with 5 items.

1) In fact

$$
\frac{1}{n^{s}} \Pi\left(\frac{s}{2}-1\right) \pi^{s / 2}=\int_{0}^{\infty} \mathrm{e}^{-n n \pi x} x^{(s / 2)-1} \mathrm{~d} x
$$

So when one sets

$$
\sum_{1}^{\infty} \mathrm{e}^{-n n \pi x}=\psi(x)
$$

it follows that

$$
\Pi\left(\frac{s}{2}-1\right) \pi^{s / 2} \zeta(s)=\int_{0}^{\infty} \psi(x) x^{(s / 2)-1} \mathrm{~d} x
$$

or, because

$$
2 \psi(x)+1=x^{-1 / 2}\left[2 \psi\left(\frac{1}{x}\right)+1\right]
$$

that

$$
\begin{aligned}
& \Pi\left(\frac{s}{2}-1\right) \pi^{s / 2} \zeta(s) \\
& =\int_{1}^{\infty} \psi(x) x^{(s / 2)-1} \mathrm{~d} x+\int_{0}^{1} \psi\left(\frac{1}{x}\right) x^{(s-3) / 2} \mathrm{~d} x+\frac{1}{2} \int_{0}^{1}\left(x^{(s-3) / 2}-x^{(s / 2)-1}\right) \mathrm{d} x \\
& =\frac{1}{s(s-1)}+\int_{0}^{\infty} \psi(x)\left(x^{s / 2-1}+x^{-(s+1) / 2}\right) \mathrm{d} x .
\end{aligned}
$$

2). I now set $s=\frac{1}{2}+i t$ and

$$
\Pi\left(\frac{s}{2}\right)(s-1) \pi^{s / 2} \zeta(s)=\xi(t)
$$

so that

$$
\xi(t)=\frac{1}{2}-\left(t t+\frac{1}{2}\right) \int_{1}^{\infty} \psi(x) x^{-3 / 4} \cos \left(\frac{1}{2} t \log x\right) \mathrm{d} x
$$

or also

$$
\xi(t)=4 \int_{1}^{\infty} \frac{\mathrm{d}\left[x^{3 / 2} \psi^{\prime}(x)\right]}{\mathrm{d} x} x^{-1 / 4} \cos \left(\frac{1}{2} t \log x\right) \mathrm{d} x .
$$

This function is finite for all finite values of $t$ and can be developed as a power series in $t t$ which converges very rapidly. 
3) Now since for values of $s$ with real part greater than 1 , $\log \zeta(s)=-\sum \log \left(1-p^{s}\right)$ is finite and since the same is true of the other factors of $\xi(t)$, the function $\xi(t)$ can vanish only when the imaginary part of $t$ lies between $\frac{1}{2} i$ and $-\frac{1}{2} i$. The number of roots of $\xi(t)$ whose real parts lie between 0 and $T$ is about

$$
=\frac{T}{2 \pi} \log \frac{T}{2 \pi}-\frac{T}{2 \pi},
$$

because the integral $\int \mathrm{d} \log \xi(t)$ taken in the positive sense around the domain consisting of all values whose imaginary parts lie between $\frac{1}{2} i$ and $\frac{1}{2} i$ and whose real parts lie between 0 and $T$ is (up to a fraction of the order of magnitude of $1 / T)$ equal to $[T \log (T / 2 \pi)-T] i$ and is, on the other hand, equal to the number of roots of $\xi(t)=0$ in the domain multiplied by $2 \pi i$.

4) One finds in fact about this many real roots within these bounds and it is very likely that all of the roots are real. One would of course like to have a rigorous proof of this, but I have put aside the research for such a proof after some fleeting vain attempts, because it is not necessary for the immediate objection of my investigation.

5) If one denotes by $\alpha$ the roots of the equation $\xi(\alpha)=0$, then one can express $\log \xi(t)$ as

$$
\sum \log \left(1-\frac{t t}{\alpha \alpha}\right)+\log \xi(0)
$$

because, since the density of roots of size $t$ grows only like $\log (t / 2 \pi)$ as $t$ grows, this expression converges and for infinite $t$ is only infinite like $t \log t$; Thus it differs from $\log \xi(t)$ by a function of $t t$ which is continuous and finite for finite $t$ and which, when divided by $t t$, is infinitely small for infinite $t$. This difference is therefore a constant, the value of which can be determined by setting $t=0$.

Remark. 1) It is strange that Riemann had not given a complete integral expression (1.6) of $\xi(s)$. I think this is a neglect, which will bring misunderstanding later. 2) He took $s=1 / 2+i t$ and derived a complete expression $\xi(t)$, which is an even real function and $\operatorname{Im}(\xi)=0$, this is an important symmetry. 3) Riemann had a conjecture on the number $N(T)$ of zeros (proved by Mangoldt, 1905). This estimate was applied to the product expression. 4) The RH is the greatest mystery. Later Siegel (1932) had found a computational formula unpublished in Riemann's manuscript (now called Riemann-Siegel formula, which is still to discuss $\xi$ ) and the first several zeros computed. Siegel had quite surely pointed out that his manuscript had not any steps to go to proof of RH. 5) Riemann had proposed another conjecture on the product expression of $\xi$ (proved by Hadamard, 1893), which is the base to prove the positivity by Lagarias(1999).

Now, when RH is already proved, we would make the following three com- 
ments:

1) In these 5 items, Riemann had always discussed $\xi$, rather than $\zeta$. I don't know that from what time, $\mathrm{RH}$ had become to study $\zeta$. This has gone against the original thinking of Riemann and was a historic misguide. Maybe, there is a possibility that due to the decay of $\xi$, computing $\xi$ is too hard, while computing $\zeta$ is easier, but analysis method for $\zeta$ is hopeless. Actually, a unique hope is to study $\xi$.

2) Except for local PVS to be proposed by us, Riemann had already prepared the main tools needed in proving $\mathrm{RH}$, e.g. the entire function $\xi(s)$, the symmetry, The number of zeros and the product expression and so on. What wise Riemann was!

3) How to find RH? I think that Riemann had proposed RH likely based on the finite numerical results and theoretical consideration for the symmetry. In my opinion, Riemann's thinking, really, looks a little like to the eastern mathematical thinking. Fortunately, I have detected the essence of $\xi(s)$ and proved RH by use of Liuhui methodology, see $\S 7$.

Finally we recall several key progressions along Riemann thinking as follows.

Riemann (1859) constructed $\xi$ and proposed several conjectures, of key RH;

Hadamrd (1893) proved the product formula of $\xi$;

Mangoldt (1905) proved a number $N(T)$ of zeros of $\xi$;

Siegel (1932) found R-S formula on critical line for $\xi$;

Lagarias (1999) found and proved the positive $\operatorname{Re}\left(\xi^{\prime} / \xi\right)>0$ outside critical line;

Author (2020) found PVS and proved RH by local geometric analysis for $\xi$.

\section{Why to Give Up $\zeta ?$}

It is known that $\zeta$ has $2 \mathrm{~m}$-order Euler-Maclaurin evaluation [4]

$$
\begin{aligned}
\zeta(s)= & \sum_{n=1}^{N-1} n^{-s}+\frac{N^{1-s}}{s-1}+\frac{1}{2} N^{-s}+\frac{B_{2}}{2} s N^{-s-1}+\cdots \\
& +\frac{B_{2 m}}{(2 m) !} s(s+1) \cdots(s+2 m-2) N^{-s-2 m+1}+R_{2 m},
\end{aligned}
$$

with the remainder

$$
R_{2 m}=-\frac{s(s+1) \cdots(s+2 m-1)}{(2 m) !} \int_{N}^{\infty} B_{2 m}(\{x\}) x^{-s-2 m} \mathrm{~d} x
$$

where $B_{i}(x)$ is $i^{\text {th }}$ Bernoulli polynomial, $B_{i}$ is $i^{\text {th }}$ Bernoulli number and $\{x\}$ is fractional part of $x$. It is an analytic continuation and the most efficient computing formula up to now. Clearly, it is impossible to prove $\mathrm{RH}$ by so complicated double series.

Taking $2 m=10$ and $N \geq 10 t$ and neglecting its remainder, the desired accuracy can be attained. Denote $\zeta(s)=U+i V$. Figure 7 exhibits $U$ (real line) and $V$ (dot line) (and their absolute values) on critical line $9990<t<9995$ and 6 roots are found. Their structure near zeros is very different from $\xi$ : 
1). $U$ and $V$ are of same order, even if on critical line, $V$ does not disappear, no symmetry.

2). $\zeta$ behaves badly near zeros, sometimes $U$ and $V$ are almost tangent. What geometry structure it is? No PVS.

To draw curves, we use the norm $|\zeta(s)|=|U|+|V|$. Edwards [5] (p. 178-179) had discussed two roots("near" double zero) $t_{1}=17143.786536$ and $t_{2}=17143.821844$ for $\beta=0$, and $t_{2}-t_{1}=0.035308$ is very small, see the left of Figure 8. He wrote that: "there is no obvious reason why the exception to this statement could not include a counterexample to the Riemann hypothesis". I think that he had considered only approximation of the infinite series, while neglected the property of $\zeta$ itself. Actually, we have computed this case, see the right of Figure 8, its lower bound $\min _{t}|\zeta|>0$ monotone increases for
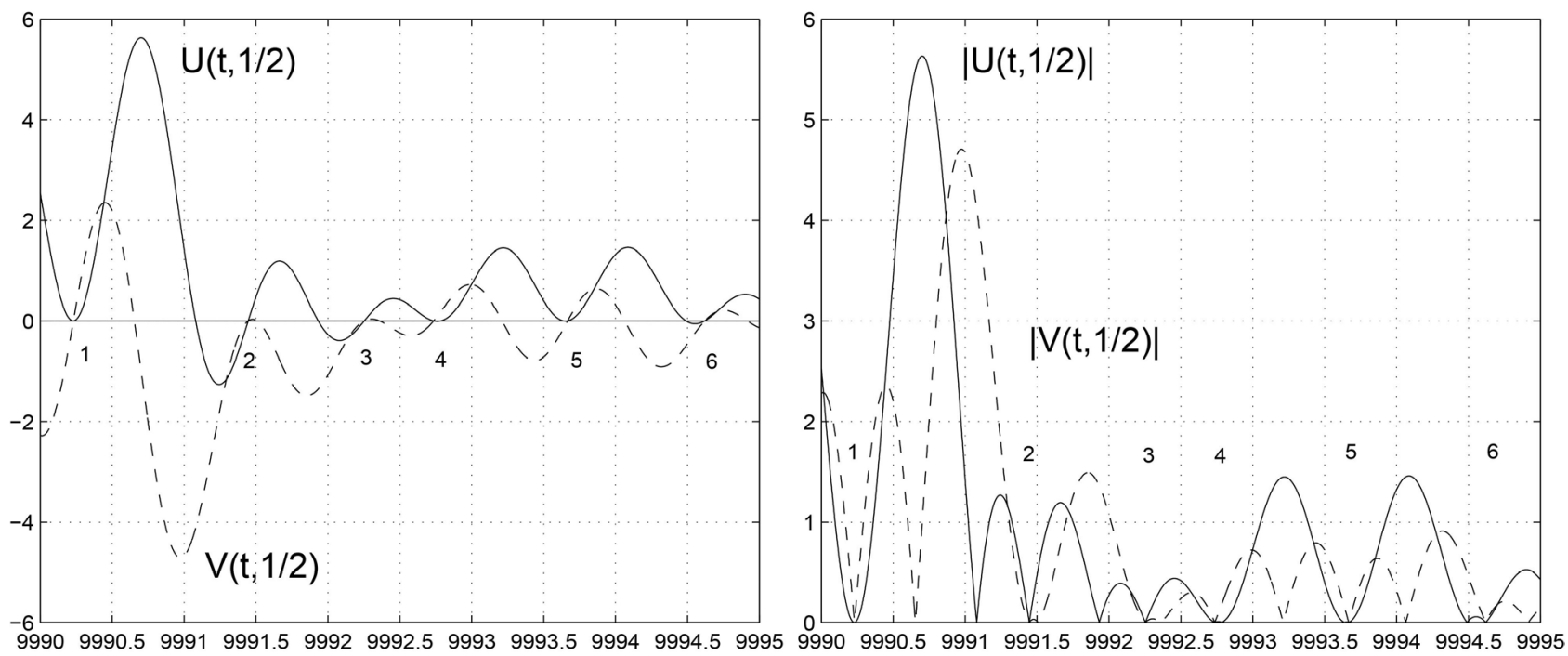

Figure 7. $U$ (real line) and $V$ (dot line) on critical interval $t \in(9990,9995)$.
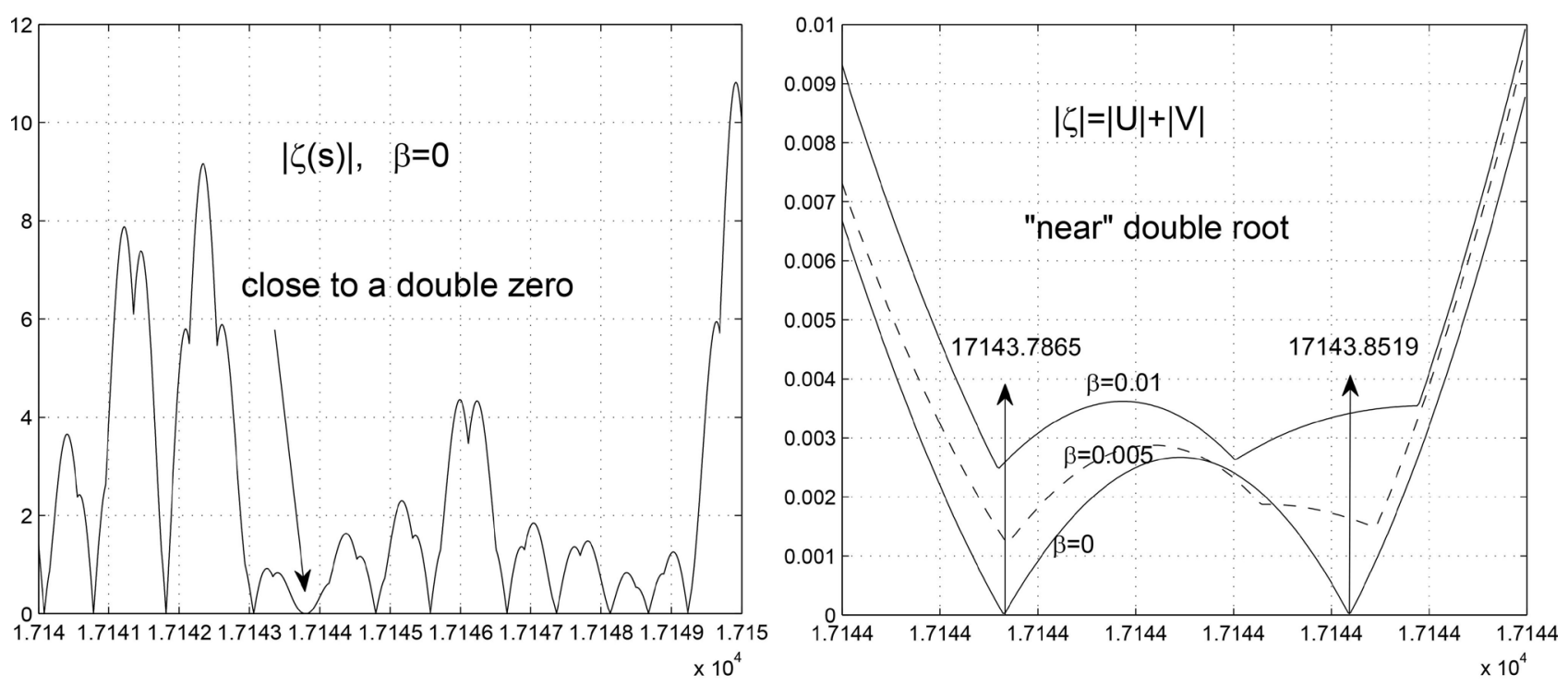

Figure 8. Growth of "near" double roots for $\beta=0,0.005,0.01$. 
$\beta=0.005,0.01$. This is a mystery to be hidden behind $\zeta$, which consists with theorem 3 .

\section{Guide Role of Liuhui Thinking in Proving RH}

Greek preferred the deduction. To face so difficult $\mathrm{RH}$, where did the idea of proof come from? The Greek mathematics does not give any inspiration. But the eastern mathematics may do it! Because Chinese emphasized the combination of computing and analyzing, in particular, Liuhui thought that "can detect the unknown and method by computing". We shall show how to reveal the essence of RH by Liuhui methodology.

\subsection{Liuhui Was the Greatest Mathematician in Ancient China}

Chinese "Nine Chapters Mathematics" (at least B.C.4-2 century) and Greek "Geometry Original" (B.C. 3-2 century) are two mathematical classics over the world, and also are different systems of mathematical idea and method. Liuhui (A.D.225-295) made about one hundred remarks in "Nine Chapters" (A.D.263) [13], and basically formed a mathematical system including geometry, computation, algebra and analysis. By analyzing these remarks, we found that his deep idea had already surpassed that period so that cannot be understood by posterity, and forgotten about 1500 years. Up to recent 50 years, it is gradually recognized newly [14]. We list five items of Liuhui's discovery as follows.

\section{1) Sum and take limit to prove the existence of $\pi$.}

In B.C.11 century, Shanggao theorem (Pythagoras theorem, in B.C. 5 century) was proposed and extensively applied. Liuhui in "cut circle" (1600 words. English version [15]) had computed the area of 96-polygons to get $\pi_{96}=3.14$ (the area is more intuitive than the circumference) and then $\pi_{3072}=3.1416$. He for first time had proposed the limit concept:

"The more finely is cut, the less loss there is. Cut it again and again until one is unable to cut further, that is, when the shape coincides with that of the circle and there is no loss".

He introduced a small square $\mathrm{T}$ (rather than circumscribed polygons) and proved the existence of $\pi$ by pressing of both sides, see Figure 9

$$
\pi_{2 n}=\pi_{n}+\sum T / 2<\pi<\pi_{n}+\sum T=2 \pi_{2 n}-\pi_{n}, \text { i.e. linear interpolation }
$$

Although Archimedes computed the circumference $\pi_{96}=3.14$, Dauben [16] pointed out that he used "exhaustion", no limit concept. Walis proposed the limit concept in 1655.

2) Discover extrapolation (Richardson proposed extrapolation in 1927).

Liuhui considered the ratio of two small squares (found by Wang [17], 1996)

$$
r_{12}=\frac{\pi_{24}-\pi_{12}}{\pi_{48}-\pi_{24}}=3.95, \text { theoretical value } 4
$$

and got the extrapolation value $\pi_{192}^{*}=\pi_{192}+\left(\pi_{192}-\pi_{96}\right) /\left(r_{12}-1\right)=3.1416$. But he felt anxious, finally computed $\pi_{3072}=3.1416$ and said "the ratio obtained is 


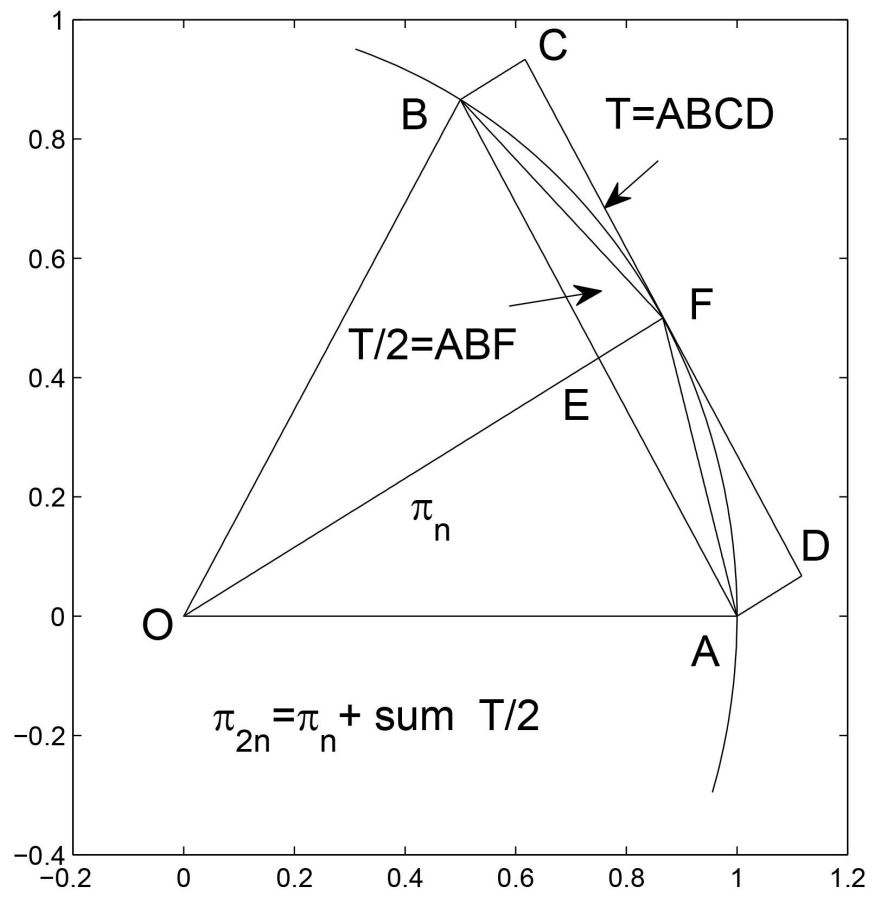

Figure 9. A small "square" $\mathrm{T}=\mathrm{ABCD}$ and role of $T / 2$.

the same as the previous one, in this way, the ratio is again verified'. This is the prediction-correction idea.

The extrapolation is an important idea of modern scientific computation. We [18] proposed the extrapolation prediction multiple grid method to solve PDE.

3) New elimination method for linear system of equation.

He used the arrangement (like the present augmented matrix) and elimination. This was a classical algorithm in ancient China (Gauss elimination appeared in 18 century).

4) Compute the area and volume, the seed of defined integral.

Liuhui proposed the "irreducible method" and limit to prove the tetrahedron volume $V=\frac{1}{3} S H$. After 200 years, Zuchongzi (A.D. 429-500) proved the sphere volume $V=\frac{4}{3} \pi R^{3}$ by this method. The both play an important role in completing Chinese mathematical system (although Archimedes has already obtained). While Liu-Zu principle, "the area and hight are same, then their volume is also same", called Cavalieli principle (1635) in the west.

5) Use "rate" and "multiple difference" to study the ratio of difference, the seed of the "slope". Ancient Greek did not have these concepts.

"Nine Chapters" discussed practical problems, the "relation" of two quantities just is function, and the piecewise expression of function was used. While the "rate" (called "lv" in ancient China) is difference ratio of function (averaging slope or velocity) $K(x)=\frac{f(x)-f(a)}{x-a}$. e.g. $f(x)=A x+B$ has the rate $A$. The linear interpolation was extensively applied in "Nine Chapters". Fibonacci 
in "Calculus classic" (1202) had one chapter "Chida algorithm", i.e. linear interpolation, Chida just is China. Liuhui supplemented "multiple difference" as Chapter 10 in "Nine Chapters".

Multiple difference is a difference ratio of several values.

e.g. $y=A x^{2}+B$ has the rate $K(x)=A(x+a)$. But using bi-section method and three data(called "thrice watchings" in ancient China) $y_{1}=A h^{2}+B$, $y_{2}=A(h / 2)^{2}+B, \quad y_{3}=A(h / 4)^{2}+B$. One gets their differences $y_{1}-y_{2}=A\left(h^{2}-h^{2} / 4\right), \quad y_{2}-y_{3}=A\left(h^{2} / 4-h^{2} / 16\right)$, and multiple difference(now called mathematical invariant)

$$
\frac{y_{1}-y_{2}}{y_{2}-y_{3}}=\frac{1-1 / 4}{1 / 4-1 / 16}=4 \text {. }
$$

which just is Liuhui's extrapolation coefficient $r=4$. We [18] adapted the prediction $y_{3}=\left(5 y_{2}-y_{1}\right) / 4$ and conjugate gradient method (CG, as a correction) to solve PDE.

We have seen that Chinese ancient mathematical thinking was very different from Greek. In that period, Liuhui had studied these two "infinitesimal processes". But nobody could understand his thinking 50 years ago.

\subsection{Liuhui Methodology (Historic Contribution)}

Liuhui's preface ( 800 words) is a wonderful paper through ages, and expounded his deep mathematical thinking.

1) "Scientific discovery is a recognition process of the prediction and correction". Liuhui's extrapolation is a typical prediction method.

2) Mathematical methodology: "Computing can distinguish tiny and detect the unknown and method". "And analyze the reason by logic, explain the essence by figures".

This is an open, progressive and creative methodology (do not stipulate conclusion in advance), very different from Greek idea. Chinese academician Z. C. Shi pointed out [19]: "Scientific computation, experiments and theory, like a tripod, supplement each other, and become three methodologies in modern science actions", and emphasized: "Scientific computation not only is a numerical method, but also a method of research". Liuhui's idea just is third method, which plays an important guide role in studying modern mathematics [14].

When study a difficult problem, because for lack of understanding, we should detect its properties by computing in various directions, then analyze these results and look for key properties, finally find the idea of proof. This just is Liuhui thinking.

\subsection{Detect the Essence of RH by Liuhui Thinking}

I studied ancient Chinese mathematical thinking for 10 years. I believe that $\mathrm{Li}$ uhui methodology has powerful vitality. How to detect the mystery of RH? During four years, my recognition process is as follows.

1) Studying $\zeta$ is hopeless, see $\S 6$. A unique hope is to study $\xi$. 
2) We found that the real and image parts of $\xi$ have the positive phase-difference, which implies RH. But we don't know how to describe it. A chance of local geometry analysis is missed. At that time we always wanted to use the asymptotic analysis.

3) To attain the exponential decay $O\left(\mathrm{e}^{-t \pi / 4}\right)$, we used Riemann technique of integration by parts and Jacobi equality to get high-order expression, $m=2 k+2$,

$$
\left\{\begin{array}{l}
\xi(\tau)=(-1)^{k} P_{k}^{-1}(\tau)\left(W_{m}^{+}(\tau)+W_{m}^{-}(\tau)\right), \tau=\beta+i t \\
W_{m}^{ \pm}(\tau)=K^{ \pm}\left(f_{m}\right)=\int_{1}^{\infty} x^{ \pm \tau / 2} x^{-3 / 4} f_{m}(x) \mathrm{d} x, \\
P_{k}(\tau)=\prod_{j=0}^{k}(\tau / 2+1 / 4+j)(-\tau / 2+1 / 4+j), \\
f_{m}(x)=\sum_{n=1}^{\infty} \sum_{j=k+1}^{m} d_{m, j}\left(-n^{2} \pi x\right)^{j} \mathrm{e}^{-n^{2} \pi x}
\end{array}\right.
$$

where the coefficients $d_{m j}$ are defined by recurrence formula. For any $t$ we can look for suitable $m$ such that $P_{k}^{-1}(\tau) \approx O\left(\mathrm{e}^{-t \pi / 4}\right)$. Thus need not cancel each other in the integral, while directly use asymptotic analysis. But $f_{m}(x)$ for large $m$ is too complicated. We adapted the incomplete gamma function, Laplace integral and saddle point method etc., all fail.

4) We considered the integrals $W^{ \pm}(\tau)$ along complex lines $z=1+(a \pm i b) r$, and found that $f_{m}(z)$ condenses into a solitary high peak, I'm excited for this. We have constructed a simple function $g_{m}(z)=C z^{A} \mathrm{e}^{-B z}$ to simulate the high peak with relative residue $1 / 80$, where $A, B$ and $C$ are some constants independent of $\tau$, and $K^{ \pm}\left(g_{m}\right)$ can be approximately expressed by Gamma function, as a dominated function. When $t \leq 100$, using a comparison criterion, it seems $\mathrm{RH}$ is proved. But for large $t$, the asymptotic analysis is also hopeless.

5) In these researches, we have always met a wide gap: How to prove no zero for the infinite series? It is impossible. Finally, in 2019 Oct.2, we have suddenly waked up that

Give up the summation process, study the geometry structure of $\boldsymbol{\xi}$ itself.

Actually we come back to the positive phase-difference once again. But now, we have found the local peak-valley structure and the importance of symmetry, and then proved RH by geometry method, where $t$ is arbitrary.

\section{Acknowledgements}

The author expresses sincere thanks to the reviewer for his valuable comments, suggestion and kind encouragement.

\section{Conflicts of Interest}

The author declares no conflicts of interest regarding the publication of this paper.

\section{References}

[1] Smale, S. (1998) Mathematical Problems for Next Century. The Mathematical Intelligencer, 20, 7-15. 
[2] Bombieri, E. (2000) Problems of the Millennium: The Riemann Hypothesis.

[3] Conrey, J. (2003) The Riemann Hypothesis. Notices of the AMS, 50, 341-353.

[4] Borwein, P., Choi, S., Rooney, B. and Weirathmuller, A. (2008) The Riemann Hypothesis. Springer, New York. https://doi.org/10.1007/978-0-387-72126-2

[5] Edwards, H.M. (2001) Riemann's Zeta Function. Dover Publication Inc., Mineola.

[6] Durmagambetrov, A. (2016) The Riemann Hypothesis-Millennium Prize Problem. Advances in Pure Mathematics, 6,915-920. https://doi.org/10.4236/apm.2016.612069

[7] Lune, J. and Riele, H. (1983) On the Zeros of the Riemann Zeta Function in the Critical Strip. Part 3. Mathematics of Computation, 41, 759-767. https://doi.org/10.1090/S0025-5718-1983-0717719-3

[8] Lune, J., Riele, H. and Winter, D. (1986) On the Zeros of the Riemann Zeta Function in the Critical Strip. Part 4. Mathematics of Computation, 46, 667-681. https://doi.org/10.1090/S0025-5718-1986-0829637-3

[9] Lagarias, J. (1999) On a Positivity Property of the Riemann $\xi$-Function. Acta Arithmetica, 89, 213-234. https://doi.org/10.4064/aa-89-3-217-234

[10] Chen, C.M. (2020) The Symmetry of Riemann $\xi$-Function. Advances in Pure Mathematics, 10,464-470. https://doi.org/10.4236/apm.2020.108028

[11] Haglund, J. (2011) Some Conjectures on the Zeros of Approximates to the Riemann $\Xi$-Function and Incomplete Gamma Functions. Central European Journal of Mathematics, 9, 302-318. https://doi.org/10.2478/s11533-010-0095-3

[12] Sarnak, P. (2004) Problems of the Millennium: The Riemann Hypothesis.

[13] Guo, S.C. (1990) Nine Chapters Mathematics (in Chinese). Liaoning Education Press, Shenyang.

[14] Chen, C.M. (2016) New Recognition for Liu Hui's Mathematical Idea-Memory to Liu's Method of Circle Measurement for 1753-th Anniversary (in Chinese). Mathematics in Practice and Theory, 46, 274-287.

[15] Lam, L.Y. and Ang, T.S. (1986) Circle Measurements in Ancient China. History of Mathematics, 13, 325-340. https://doi.org/10.1016/0315-0860(86)90055-8

[16] Dauben, J. (2010) Archimedes and Liu Hui on Circles and Spheres. Ontology Studies, 10, 21-38.

[17] Wang, N.C. (2003) "Cut Circle”, Thinking through Ages-Liuhui's Big Wisdom (in Chinese). 2nd Edition, Huazhong University of Science and Technology Press, Wuhan.

[18] Chen, C.M., Shi, Z.C. and Hu, H.L. (2011) Extrapolation Cascadic Multigrid Method. Journal of Computational Mathematics, 29, 684-697. https://doi.org/10.4208/jcm.1110-m11si05

[19] Shi, Z.C. (2000) The Third Science Method-Scientific Computation in Computer Period. Qinghua University Press and Jinan University Press, Beijing and Jinan. (in Chinese) 